\title{
Cystic Fibrosis and Sleep Circadian Rhythms
}

\author{
Mariam Louis · Peter Staiano $\cdot$ Lavender Micalo $\cdot$ Nauman Chaudary
}

Received: December 22, 2021 / Accepted: January 24, 2022 / Published online: February 11, 2022

(C) The Author(s) 2022

\begin{abstract}
Cystic fibrosis (CF) is due to a mutation in the cystic fibrosis transmembrane conductance regulator gene (CFTR), which leads to unusual water and chloride secretion across epithelial surfaces. The lungs are responsible for most morbidity, though other organs are frequently affected. Sleep abnormalities have long been recognized in CF. Abnormal ventilation and oxygenation, sinus disease, deconditioning due to muscle weakness and recurrent infections, and inflammation have been thought to play a role in sleep disorders in CF. However, there is evidence that CFTR gene dysregulation can affect circadian rhythms in CF. Early recognition and treatment of circadian rhythms may improve outcomes in CF.
\end{abstract}

M. Louis · P. Staiano

Department of Pulmonary, Critical Care and Sleep Medicine, University of Florida, Jacksonville, FL, USA

L. Micalo $\cdot$ N. Chaudary $(\bowtie)$

Division of Pulmonary Disease and Critical Care Medicine, Department of Medicine, Adult Cystic Fibrosis Center, Virginia Commonwealth

University, 1200 East Broad Street, Box 980050,

Richmond, VA 23298, USA

e-mail: nauman.chaudary@vcuhealth.org
Keywords: Cystic fibrosis; CFTR dysregulation; Circadian rhythm; Sleep disorders; Sleep quality

\section{Key Summary Points}

Poor sleep quality has been known to have a negative impact on one's quality of life.

Evidence suggests that cystic fibrosis (CF) patients may have circadian disturbances, ultimately affecting sleep quality.

Understanding the implications of poor sleep quality in this population is crucial in managing CF patients.

This study illuminates a potential link between CFTR dysfunction and circadian rhythms, which may lead to new treatment strategies for circadian rhythm sleep disorders in CF patients.

\section{INTRODUCTION}

All organisms have circadian rhythms that follow a 24-h (circadian) pattern, independent of environmental cues from single-cell organisms to humans [1]. In humans, circadian rhythms are expressed in all tissues and are regulated centrally by the suprachiasmatic nucleus (SCN) 
of the anterior hypothalamus [2]. The SCN is, in turn, regulated by peripheral clocks that have circadian rhythms, also known as zeitgebers. Zeitgebers include light, mealtimes, and social interactions, which affect the underlying circadian timing [1].

Circadian patterns are critical for sleep health and optimal functioning of other organ systems [1]. Desynchrony between our internal circadian timing system and desired sleep-wake times can disrupt many physiological systems, including, but not limited to, daily rhythms in core body temperature, cortisol, appetite, immunity, and sleeping patterns [3]. Evidence suggests that patients with CF may have intrinsic circadian disturbances resulting in dysfunctional gene regulations that may affect the circadian clock and, ultimately, sleep quality.

Poor sleep quality has been associated with increased mortality risk and susceptibility to infections such as the rhinovirus in non-CF adult patients without CF [5]. Despite the importance of sleep quality, few studies have investigated the risk factors of circadian rhythms sleep disorders in the CF population [4]. Furthermore, the screening and optimal treatment strategies for circadian rhythm sleep disorders in CF patients have proven challenging in the clinic [5].

To address this paucity in the literature, we reviewed publications on sleep circadian rhythms in CF populations featured on PubMed, Elsevier, and Google Scholar up until 2021. Keywords 'cystic fibrosis', 'CFTR', and 'circadian rhythms' were used to search for relevant publications. We also manually identified studies investigating CFTR dysfunction pertaining to circadian rhythms and those that also looked at circadian rhythm disorders in CF patients. This paper will review the available literature on $\mathrm{CF}$ and sleep circadian rhythms and explore the potential link between CFTR dysfunction and misaligned circadian rhythms. This article is based on previously conducted studies and does not contain any new studies with human participants or animals performed by any of the authors.

\section{NORMAL CIRCADIAN PATTERNS}

Circadian clocks are driven by transcriptional and translational feedback loops that oscillate so that each clock can maintain a 24-h rhythm [6]. Melatonin, a potent neurohormone, is regulated by the SCN and can feedback and adjust SCN activity and is also affected by genetics $[7,8]$. A 24-h feedback loop that contains both positive elements (the CLOCK and BMAL genes) and negative elements (PER and CRY genes) regulates the rhythmic patterns of the circadian clock $[6,9]$. Mutations in any step of this process can alter the timing and length of the circadian clock $[2,3]$.

Zeitgebers, also known as time givers, help reset the circadian clock each day. Light is the most potent zeitgeber in humans [9]. Exposure to light in the early biological evening will delay the circadian clock, while exposure during the latter portion of the night advances the circadian clock [10]. Light from the retinal ganglion cells sends inputs to the SCN, which in turn sends signals to the different regions of the hypothalamus that control rhythmic behaviors (sleep-wake cycle), feeding, core body temperature, and hormonal release [8].

These circadian patterns shift somewhat throughout a person's lifespan [3]. Circadian rhythms gradually emerge between birth and the first several months of life and consolidate [9]. Children tend to have an early chronotype during early childhood but then shift to a late chronotype during adolescence (night owls) [3]. This delay in the circadian pattern gradually advances in adulthood and the elderly (early birds) [3].

Synchrony between a person's natural circadian pattern and the social timing of sleep is needed to maintain optimal sleep and organ functions [11]. In a recent review, Hou et al. report that circadian disruption is associated with adverse cardiovascular, metabolic, psychiatric, pro-oncological, and neurogenerative consequences [12]. There is also a growing body of evidence suggesting that disturbances in circadian clocks can lead to increased susceptibility to infections [13] and that crosstalk between circadian rhythms and microbiota exist [14]. 
In the next section, we will review some of the well-known disorders of circadian patterns in CF patients.

\section{CIRCADIAN RHYTHM SLEEP DISORDERS IN CF}

Circadian rhythm sleep disorders (CRSDs) are a group of sleep-wake disorders that arise from an altered endogenous circadian system [15]. Patients diagnosed with CRSDs are known to be unable to sleep when expected or needed [11]. The International Classification of Sleep Disorders, Third Edition (ICSD-3) has identified four intrinsic (delayed sleep-wake phase disorder, advanced sleep-wake phase disorder, non-24-h sleep-wake rhythm disorder, and irregular sleep-wake rhythm disorder) and two extrinsic or behavioral circadian disorders (jet lag disorder and shift work disorder) (Fig. 1), [16]. However, in this review, we will focus on the delayed sleep phase disorder.

\section{Delayed Sleep-Wake Phase Disorder (DSPD)}

DSPD is the most common intrinsic disorder and has a prevalence rate of $7-16 \%$ in adolescents [10]. Patients diagnosed with DSPD have a circadian system that promotes wakefulness until late in the evening (night owls), which results in delayed sleep and wake times compared to the rest of the population, with sleep typically occurring after midnight [17], (Fig. 3). If one attempts to sleep at an earlier bedtime, sleep onset insomnia will result, and when conventional rise times are required (such as school or work), they have great difficulty waking up and feeling alert [7]. According to Weitzman and colleagues, approximately $5-10 \%$ of patients diagnosed with chronic insomnia have DSPD [18]. Studies have also demonstrated a genetic link between structural polymorphisms in the human period3 gene and DSPD [19].

While there is a paucity of studies investigating CRSDs in the CF population, new studies present a potential link between CFTR dysfunction and DSPD. Jensen et al. evaluated how CFTR dysfunction and sleep dysregulation could clinically impact CF patients by evaluating sleep patterns in CF patients compared to a healthy control group [16]. They found that CF patients suffered from later sleep onset, longer sleep latency, and later wake times (common characteristics of DSPD) than their healthy counterparts. Moreover, these findings were independent of other CF-related markers of

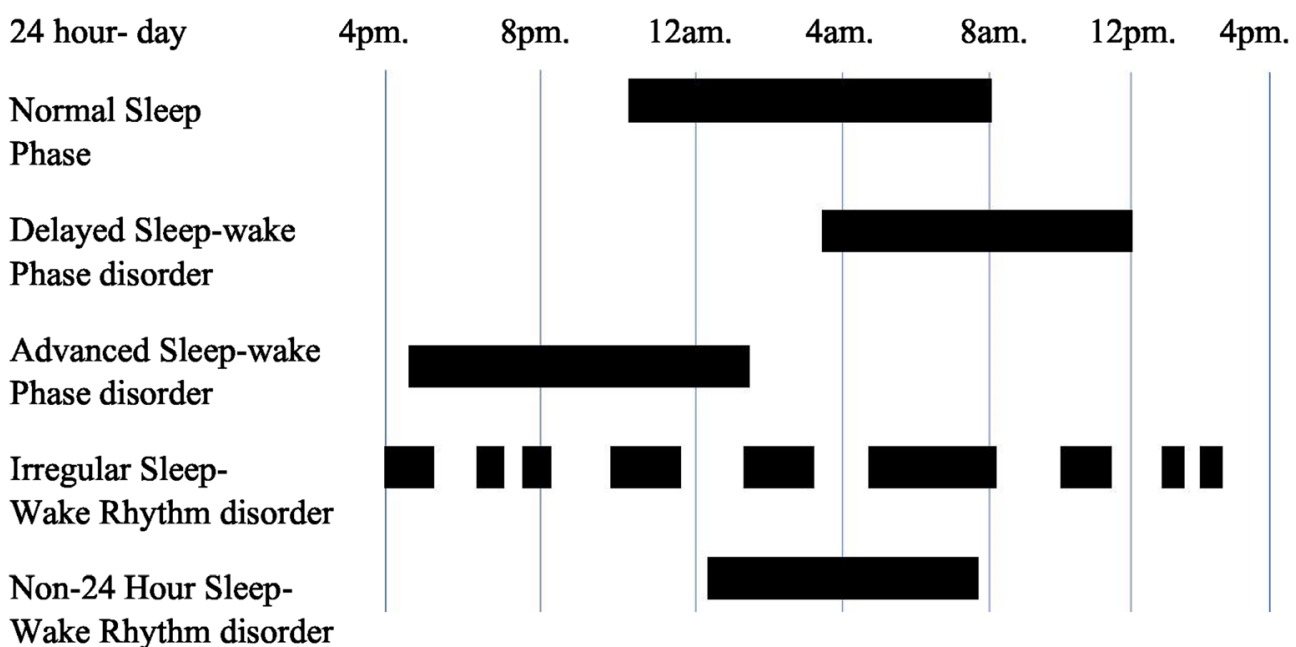

Fig. 1 A 24-h schematic sleep schedules for advanced sleep phase, delayed sleep phase, non-24-h sleep-wake rhythm, and irregular sleep-wake rhythm disorders compared to the normal sleep phase. The black-filled bars represent the sleep period. Data from these sources $[4,11,12]$ 
disease severity, such as the occurrence of CF respiratory exacerbations, predicted FEV1, and weight [20]. Thus, they assumed that this meant that the delayed sleep phase was a primary manifestation of CFTR dysfunction in CF patients [16]. Dysfunctional CFTR in the retina and anterior hypothalamus has also been associated with a misaligned circadian clock, leading to decreased quality of life and circadian delay; often associated with DSPD, in the CF population [16].

\section{PATHOPHYSIOLOGY OF CF- RELATED CIRCADIAN DISTURBANCES}

It is well established that circadian disruption can have substantial adverse effects on functionality, cognitive ability, and overall health and quality of life [20]. These effects may be even more apparent in the CF population, where several factors disrupt sleep and circadian rhythms.

\section{Impaired Sleep in CF Populations}

Studies investigating the sleep quality in CF patients report both poor objective and subjective sleep disturbances in CF patients [21]. Objective sleep quality reports focus on the total sleep time, sleep fragmentation, the number of awakenings throughout the night, and sleep architecture as estimated by the polysomnography (n-PSG) [22]. On the other hand, subjective sleep quality studies vary from objective sleep quality studies as it is measured by looking at sleep parameters such as initiation, continuity, and depth as assessed by surveys, questionnaires, or sleep logs [23].

CF patients usually report poor subjective sleep disturbances at a higher frequency, attributed to multiple factors such as nocturnal coughs, abdominal pain, gastroesophageal reflux, and side effects of their medication $[24,25]$. In a questionnaire sleep study, $63 \%$ of CF patients associated coughing with sleep disruption, which worsened with lower FEV1 values [26]. Bouka and colleagues also believed that the low CFQ18 + R (the German version of the Cystic Fibrosis Questionnaire for adults) values in the eating disturbances and digestive symptoms domains mean that gastrointestinal reflux and abdominal pain can be associated with poor subjective sleep in CF patients [20].

Though subjective poor sleep quality reports are common in CF patients, few studies report poor objective sleep quality in CF patients [21]. Amin and colleagues used actigraphy to study the correlation of disease severity (FEV1) and sleep quality in CF patients [27]. They found that CF patients had lower sleep efficiency and frequent awakenings than control patients. Lower nocturnal oxygen saturation and higher respiratory event indices have worsened sleep fragmentation in CF patients than controls [28]. A study by Meltzer and colleagues found that reduced total sleep time in CF patients may be due to treatments such as chest physiotherapy and administration of medications [29].

Although there are limited well-controlled studies assessing the impact of poor sleep on circadian rhythms in patients with CF, it is well established that sleep disruptions can negatively impact circadian rhythms in non-CF patients [3, 10, 11]. In a group study of 672 randomly chosen participants from the National Social Life, Health and Aging Project, both higher sleep fragmentation score $[\beta=0.02$; 95\% confidence interval (CI) $0.00-0.03]$ and longer wake after sleep onset $(\beta=0.27 ; 95 \% \mathrm{CI}$ 0.04-0.51) were significantly associated with higher daytime cortisol levels; a surrogate of circadian rhythms [30]. Kimura and colleagues reported that the core-body temperature in school-aged children with circadian rhythm sleep-wake disorders improved after 6-8 weeks of chronotherapy [31]. Some studies found that sleep disruption and circadian rhythm disturbances had deleterious effects on glucose and insulin hemostasis, independent of sleep duration in healthy volunteers [32, 33]. These studies illuminate the need to assess circadian rhythm disturbances in CF patients. 


\section{The CFTR Gene and Circadian Rhythms Disturbances}

It has always been widely assumed that CF-related symptoms are the primary cause of sleep disruption in CF patients, as described above [24]. However, recent studies implicate circadian rhythm disturbances and CFTR dysfunction as potential causes of sleep disturbances in CF patients [34].

Over 2000 different CFTR gene mutations have been identified [35]. Aside from the wellknown complications of ion and water transport from CFTR dysfunction (or complete nonfunction) in the CF population, there is growing evidence that poor sleep quality and sleep phase delay result from this gene mutation [36].

Barbato et al. collected tissue samples from both CF-afflicted and healthy mice counterparts and compared tissue samples obtained from both groups in both standard and sleep-deprived states [34]. They found that the CF group had a significant expression of dysregulated circadian clock genes, which they assumed was due to loss of CFTR functionality [21]. In another recent study, Barbato and colleagues associated microtubule instability with disordered circadian rhythms in CF mice [31]. $\mathrm{Ng}$ also observed that CFTR mRNA exhibits "true" circadian rhythmicity, as its rhythmic expression persists without the external cue of light (i.e., it is endogenous) [37]. This study notes that the mRNA expression of Bmal1 and Per2, two core clock genes upstream from CFTR, is also rhythmic regarding the myogenic signaling element in mice, suggesting that the CFTR may have its circadian pattern [37]. A study by Chauvet and colleagues found that lung epithelial cells with the CFTR delF508 mutation had higher PROKR2 (prokineticin 2) expression levels than normal epithelial cells [38]. Cheng and colleagues associated PROKR2 with behavioral circadian rhythm regulation in the SCN [39], suggesting a potential link between CFTR dysfunction and circadian rhythm disturbances.

In addition, CFTR appears to play an essential role in cranial nerve pathways implicated in regulating the circadian clock [40]. CFTR mRNA expression occurs in the anterior hypothalamus, which houses the circadian pacemaker
(SCN) [11]. CFTR gene dysfunction affects sodium and fluid movement across epithelial surfaces responsible for facilitating normal responses to light, which is a strong zeitgeber [41]. Work by Kim et al. found that CFTR may be implicated in phase-shifting the circadian clock via the H1R- G $\beta \gamma$-cAMP/PKA histamine pathway in the SCN neurons to activate CaV1.3 channels through CFTR-mediated $\mathrm{Cl}(-)$ efflux [42].

These data suggest that the CFTR gene may play an essential role in regulating circadian rhythms. At the same time, it is not clear if dysfunction of the CFTR may lead to alterations in circadian timing and normal physiological responses to daytime hours and school/work schedules. These alterations can have significant social and academic implications in CF patients, including difficulty succeeding in academics (especially with early morning classes) and their professional careers.

\section{PHARMACOLOGY FOR CIRCADIAN RHYTHM SLEEP DISORDERS IN CF}

Due to the negative impact poor sleep quality in CF patients has on their quality of life, understanding the implications of poor sleep quality is crucial in managing CF patients. There are two pillars of management of CRSD, namely behavioral and pharmacological. The exact therapy depends on the type of CRSD [38]. Please refer to Table 1 for a summary of the general principles of management [43].

There are limited data on specific therapeutic interventions of CRSD in CF patients, although it would be reasonable to use the general principles outlined in Table 1.

As previously mentioned, CFTR dysfunction has been associated with circadian rhythm phase delays in CF, which might mean that CFTR modulator therapy may be a promising modality. Studies evaluating CFTR modulator therapy have improved lung function and decreased exacerbations even in advanced CF stages [44]. In addition, ivacaftor, a CFTR potentiator, was shown to improve sleep-related quality of life in patients with $\mathrm{CF}$ at 1 and 3 months using the Sino-Nasal Outcome Test 
Table 1 Summary of treatment recommendations for intrinsic CRSD

\begin{tabular}{lll}
\hline & Light & Melatonin $^{\mathbf{a}}$ \\
\hline $\begin{array}{l}\text { Delayed sleep-wake } \\
\text { phase disorder }\end{array}$ & $\begin{array}{l}\text { Bright morning light } \\
\text { Avoid evening light }\end{array}$ & Strategically timed administration may be of benefit \\
$\begin{array}{c}\text { Advanced sleep-wake } \\
\text { phase disorder }\end{array}$ & $\begin{array}{c}\text { Bright light in the evening } \\
\text { may be beneficial }\end{array}$ & Limited evidence \\
$\begin{array}{c}\text { Non-24-h sleep-wake } \\
\text { rhythm disorder }\end{array}$ & No clear benefit & May be of benefit in the totally blind \\
$\begin{array}{c}\text { Irregular sleep-wake } \\
\text { rhythm disorder }\end{array}$ & No clear benefit & May be of benefit in patients with neurological disorders outside \\
\end{tabular}

${ }^{\mathrm{a}}$ Or melatonin receptor agonist. Data from Burgess and Emens [39]

questionnaire (1 month: $\quad-0.35 ; \quad P<0.01$; 3 months: $-0.32, P<0.01 ; 6$ months: -0.18 , $P=0.07$ ) [45]. Improved quality of life and rhinological and psychological domains were also reported [40]. Other pharmacotherapies in CF have targeted sleep apnea, altered sleep patterns, and sleep disturbances to improve quality of life and other outcomes [21]. Most interventions are widely considered effective in the short term, but there is limited evidence in the long term on morbidity and mortality, which proves the need for more research.

\section{FUTURE DIRECTIONS}

Circadian disturbances are likely more common than previously realized in patients with CF and warrant further investigation. The relationship between the CFTR gene and its role in regulating the circadian pattern may have clinical implications. Studies have reported alteration in circadian clock gene expression and modulation of lung function as a whole [34]. Limited information on impaired sleep's effect on CF patients' immune system, brain function, and metabolic activity warrants further research in this area.

Patients with CF may be inherently prone to lack of sleep from chronic coughing, hypoxemia, hypercapnia, treatment, and social requirements. Despite this information, CF patients are not routinely screened CF patients for sleep impairment. Existing sleep impairment data in CF are limited and often contradicting. A paucity of data also exist for treatment approaches for sleep disorders in CF. There remains an urgent need to have future studies that can address these deficiencies $[4,5]$.

\section{ACKNOWLEDGEMENTS}

Funding. No funding or sponsorship was received for this study or publication of this article.

Authorship. All named authors meet the International Committee of Medical Journal Editors (ICMJE) criteria for authorship for this article, take responsibility for the integrity of the work as a whole, and have given their approval for this version to be published. Lavender works as a research volunteer with Dr. Chaudary. Dr. Chaudary is a Professor of Medicine and Director of the Adult Cystic Fibrosis Center at Virginia Commonwealth University Medical Center.

Author Contributions. All named authors contributed equally to the writing of this manuscript. 
Disclosures. Lavender Micalo, Nauman Chaudary, Peter Staiano, and Mariam Louis have nothing to disclose.

Compliance with Ethics Guidelines. This article is based on previously conducted studies and does not contain any new studies with human participants or animals performed by any of the authors.

Open Access. This article is licensed under a Creative Commons Attribution-NonCommercial 4.0 International License, which permits any non-commercial use, sharing, adaptation, distribution and reproduction in any medium or format, as long as you give appropriate credit to the original author(s) and the source, provide a link to the Creative Commons licence, and indicate if changes were made. The images or other third party material in this article are included in the article's Creative Commons licence, unless indicated otherwise in a credit line to the material. If material is not included in the article's Creative Commons licence and your intended use is not permitted by statutory regulation or exceeds the permitted use, you will need to obtain permission directly from the copyright holder. To view a copy of this licence, visit http://creativecommons.org/licenses/by$\mathrm{nc} / 4.0 /$.

\section{REFERENCES}

1. Baron KG, Reid KJ. Circadian misalignment and health. Int Rev Psychiatry. 2014;26(2):139-54. https://doi.org/10.3109/09540261.2014.911149.

2. Kondratova AA, Kondratov RV. The circadian clock and pathology of the ageing brain. Nat Rev Neurosci. 2012;13(5):325-35. https://doi.org/10.1038/ nrn3208.

3. Reddy S, Reddy V, Sharma S. Physiology, circadian rhythm. In: StatPearls. Treasure Island: StatPearls Publishing; 2021 [Online]. http://www.ncbi.nlm. nih.gov/books/NBK519507/. Accessed 2 Dec 2021.

4. Shakkottai A, O'Brien LM, Nasr SZ, Chervin RD. Sleep disturbances and their impact in pediatric cystic fibrosis. Sleep Med Rev. 2018;42:100-10. https://doi.org/10.1016/j.smrv.2018.07.002.
5. Huang C-Y, et al. The association between zolpidem and infection in patients with sleep disturbance. J Psychiatr Res. 2014;54:116-20. https://doi.org/10. 1016/j.jpsychires.2014.03.017.

6. Zee PC, Abbott SM. Circadian rhythm sleep-wake disorders. CONTINUUM Lifelong Learn Neurol. 2020;26(4):988-1002. https://doi.org/10.1212/ CON.0000000000000884.

7. Jones CR, Huang AL, Ptáček LJ, Fu Y-H. Genetic basis of human circadian rhythm disorders. Exp Neurol. 2013;243:28-33. https://doi.org/10.1016/j. expneurol.2012.07.012.

8. Benarroch EE. Suprachiasmatic nucleus and melatonin: reciprocal interactions and clinical correlations. Neurology. 2008;71(8):594-8. https://doi. org/10.1212/01.wnl.0000324283.57261.37.

9. Logan RW, McClung CA. Rhythms of life: circadian disruption and brain disorders across the lifespan. Nat Rev Neurosci. 2019;20(1):49-65. https://doi. org/10.1038/s41583-018-0088-y.

10. Barion A, Zee PC. A clinical approach to circadian rhythm sleep disorders. Sleep Med. 2007;8(6): 566-77. https://doi.org/10.1016/j.sleep.2006.11. 017.

11. Reppert SM, Weaver DR. Coordination of circadian timing in mammals. Nature. 2002;418(6901): 935-41. https://doi.org/10.1038/nature00965.

12. Hou Y, Liu L, Chen X, Li Q, Li J. Association between circadian disruption and diseases: a narrative review. Life Sci. 2020;262: 118512. https:// doi.org/10.1016/j.lfs.2020.118512.

13. Borrmann H, McKeating JA, Zhuang X. The circadian clock and viral infections. J Biol Rhythms. 2021;36(1):9-22. https://doi.org/10.1177/ 0748730420967768.

14. Pearson JA, Wong FS, Wen L. Crosstalk between circadian rhythms and the microbiota. Immunology. 2020;161(4):278-90. https://doi.org/10.1111/ imm. 13278 .

15. Zhu L, Zee PC. Circadian rhythm sleep disorders. Neurol Clin. 2012;30(4):1167-91. https://doi.org/ 10.1016/j.ncl.2012.08.011.

16. Sateia MJ. International classification of sleep disorders-third edition. Chest. 2014;146(5):1387-94. https://doi.org/10.1378/chest.14-0970.

17. Paine S-J, Fink J, Gander PH, Warman GR. Identifying advanced and delayed sleep phase disorders in the general population: a national survey of New Zealand adults. Chronobiol Int. 2014;31(5):627-36. https://doi.org/10.3109/07420528.2014.885036. 
18. Weitzman ED. Delayed sleep phase syndrome: a chronobiological disorder with sleep-onset insomnia. Arch Gen Psychiatry. 1981;38(7):737. https:// doi.org/10.1001/archpsyc.1981.01780320017001.

19. Ebisawa T, et al. Association of structural polymorphisms in the human period3 gene with delayed sleep phase syndrome. EMBO Rep. 2001;2(4):342-6. https://doi.org/10.1093/embo-reports/kve070.

20. Bouka A, et al. Quality of life in clinically stable adult cystic fibrosis out-patients: Associations with daytime sleepiness and sleep quality. Respir Med. 2012;106(9):1244-9. https://doi.org/10.1016/ j.rmed.2012.06.010.

21. Katz ES. Cystic fibrosis and sleep. Clin Chest Med. 2014;35(3):495-504. https://doi.org/10.1016/j.ccm. 2014.06.005.

22. O’Donnell D, Silva EJ, Münch M, Ronda JM, Wang W, Duffy JF. Comparison of subjective and objective assessments of sleep in healthy older subjects without sleep complaints. J Sleep Res. 2009;18(2): 254-63. https://doi.org/10.1111/j.1365-2869.2008. 00719.x.

23. Zavecz Z, Nagy T, Galkó A, Nemeth D, Janacsek K. The relationship between subjective sleep quality and cognitive performance in healthy young adults: evidence from three empirical studies. Sci Rep. 2020;10(1):4855. https://doi.org/10.1038/ s41598-020-61627-6.

24. Fauroux B, et al. Sleep quality and nocturnal hypoxaemia and hypercapnia in children and young adults with cystic fibrosis. Arch Dis Child. 2012;97(11):960-6. https://doi.org/10.1136/ archdischild-2011-300440.

25. Flume PA, Ciolino J, Gray S, Lester MK. Patient-reported pain and impaired sleep quality in adult patients with cystic fibrosis. J Cyst Fibros. 2009;8(5): 321-5. https://doi.org/10.1016/j.jcf.2009.07.004.

26. Stenekes SJ, Hughes A, Grégoire M-C, Frager G, Robinson WM, McGrath PJ. Frequency and selfmanagement of pain, dyspnea, and cough in cystic fibrosis. J Pain Symptom Manag. 2009;38(6): 837-48. https://doi.org/10.1016/j.jpainsymman. 2009.04.029.

27. Amin R, Bean J, Burklow K, Jeffries J. The relationship between sleep disturbance and pulmonary function in stable pediatric cystic fibrosis patients. Chest. 2005;128(3):1357-63. https://doi.org/10. 1378/chest.128.3.1357.

28. Reiter J, et al. Sleep disorders in cystic fibrosis: a systematic review and meta-analysis. Sleep Med Rev. 2020;51: 101279. https://doi.org/10.1016/j. smrv.2020.101279.
29. Meltzer LJ, Beck SE. Sleep patterns in children with cystic fibrosis. Child Health Care. 2012;41(3):260-8. https://doi.org/10.1080/02739615.2012.686365.

30. Morgan E, Schumm LP, McClintock M, Waite L, Lauderdale DS. Sleep characteristics and daytime cortisol levels in older adults. Sleep. 2017. https:// doi.org/10.1093/sleep/zsx043.

31. Kimura S, Takaoka Y, Toyoura M, Kohira S, Ohta M. Core body temperature changes in school-age children with circadian rhythm sleep-wake disorder. Sleep Med. 2021;87:97-104. https://doi.org/10. 1016/j.sleep.2021.08.026.

32. Toyoura M, Miike T, Tajima S, Matsuzawa S, Konishi Y. Inadequate sleep as a contributor to impaired glucose tolerance: a cross-sectional study in children, adolescents, and young adults with circadian rhythm sleep-wake disorder. Pediatr Diabetes. 2020;21(4):557-64. https://doi.org/10.1111/pedi. 13003.

33. Stamatakis KA, Punjabi NM. Effects of sleep fragmentation on glucose metabolism in normal subjects. Chest. 2010;137(1):95-101. https://doi.org/ 10.1378/chest.09-0791.

34. Barbato E, Mianzo H, Litman P, Darrah R. Dysregulation of circadian rhythm gene expression in cystic fibrosis mice. J Circadian Rhythms. 2019;17(1):2. https://doi.org/10.5334/jcr.175.

35. Claustres $\mathrm{M}$, et al. CFTR-France, a national relational patient database for sharing genetic and phenotypic data associated with rare CFTR variants. Hum Mutat. 2017;38(10):1297-315. https://doi. org/10.1002/humu.23276.

36. Jensen JL, Jones CR, Kartsonaki C, Packer KA, Adler FR, Liou TG. Sleep phase delay in cystic fibrosis. Chest. 2017;152(2):386-93. https://doi.org/10. 1016/j.chest.2017.03.057.

37. Ng CH-Y. The cystic fibrosis transmembrane conductance regulator as a potential link between the circadian clock and the myogenic response. Thesis. University of Toronto. 2018.

38. Chauvet S, et al. EG-VEGF, BV8, and their receptor expression in human bronchi and their modification in cystic fibrosis: impact of CFTR mutation (delF508). Am J Physiol Lung Cell Mol Physiol. 2015;309(3):L314-322. https://doi.org/10.1152/ ajplung.00382.2014.

39. Cheng MY, et al. Prokineticin 2 transmits the behavioural circadian rhythm of the suprachiasmatic nucleus. Nature. 2002;417(6887):405-10. https://doi.org/10.1038/417405a. 
40. Blume C, Garbazza C, Spitschan M. Effects of light on human circadian rhythms, sleep and mood. Somnologie. 2019;23(3):147-56. https://doi.org/10. 1007/s11818-019-00215-X.

41. Liou TG. The clinical biology of cystic fibrosis transmembrane regulator protein. Chest. 2019;155(3):605-16. https://doi.org/10.1016/j. chest.2018.10.006.

42. Kim YS, et al. Histamine 1 receptor-G $\beta \gamma$-cAMP/PKACFTR pathway mediates the histamine-induced resetting of the suprachiasmatic circadian clock. Mol Brain. 2016;9(1):49. https://doi.org/10.1186/ s13041-016-0227-1.
43. Burgess HJ, Emens JS. Drugs used in circadian sleep-wake rhythm disturbances. Sleep Med Clin. 2020;15(2):301-10. https://doi.org/10.1016/j.jsmc. 2020.02.015.

44. Shteinberg M, Taylor-Cousar JL. Impact of CFTR modulator use on outcomes in people with severe cystic fibrosis lung disease. Eur Respir Rev. 2020;29(155): 190112. https://doi.org/10.1183/ 16000617.0112-2019.

45. McCormick J, et al. Ivacaftor improves rhinologic, psychologic, and sleep-related quality of life in G551D cystic fibrosis patients: improved quality of life in G551D CF patients. Int Forum Allergy Rhinol. 2019;9(3):292-7. https://doi.org/10.1002/alr. 22251. 